\title{
Quantitative Multivariate Analysis with Artificial Neural Networks
}

\author{
Chii-Wann Lin ${ }^{1}$, Tzu-Chien Hsiao ${ }^{2}$, Mang-Ting Zeng ${ }^{2}$, Hue-Hua Chiang ${ }^{2}$ \\ ${ }^{1}$ Center for Biomedical Engineering, College of Medicine, National Taiwan University \\ Taipei, Taiwan, R.O.C. \\ ${ }^{2}$ Institute of Biomedical Engineering, National Yang-Ming University \\ Taipei, Taiwan, R.O.C.
}

\begin{abstract}
1/4Abstract: Quantitative interpretation of spectra can be achieved by using artificial neural networks with multi-layer architecture. Both back-propagation (BP) and radial basis function ( $R B F$ ) are implemented and tested with raw absorption spectra and normalized spectra of glucose solutions in MATLAB. Simulation results showed partial least square (PLS) method can have better performance with small number of calibration set. However, with increasing size of data set as in cross validation method, RBF and BP have better performance. With optimal spreading factor, RBF can have the same degree of accuracy but significantly faster convergent speed comparing to BP. Normalization scheme can also significantly affect the performance of both RBF and BP.
\end{abstract}

\section{INTRODUCTION}

The use of spectra for chemometric measurement is always an important area for analytical chemistry and biomedical assay $[1,2]$. While the optical signals from biomedical samples can reveal information of the probed subjects, quantitative measurement with bio-optical spectral signal often requires a more robust method for faster multi-variate calibration and evaluation [3,4]. Artificial neural networks (ANN) with combined power of nonlinear processing elements (neurons) and learning capability have been used for possible handling of nonlinear and noisy data set [5]. It has been clear that multilayer neural networks with back-propagation (BP) weight update method and generalized delta rule (GDR) share the same fundamental principle as least mean square approach with iterative error minimization. The drawbacks of BP method are its slow convergent speed and number of hidden nodes for needed storage capacity. It has been shown that radial basis function (RBF) networks with optimal network parameters are able to approximate any continuous functions $[6,7,8]$. Therefore, we use a RBF network as an alternative modeling method to compare the results of different methods. Intuitively, both methods fit an internal calibration curve from the limited available input/output pairs, which often occurs in biomedical applications.

\section{METHOD}

For BP method, output neuron $\mathrm{O}_{j}$ is calculated from the weight $\left(\mathrm{W}_{\mathrm{ij}}\right)$ sum of previous layer output $\left(\mathrm{O}_{\mathrm{i}}\right)$ and bias $\left(\mathrm{B}_{\mathrm{j}}\right)$.

$$
\mathrm{O}_{\mathrm{j}}=\mathrm{F}\left(\Sigma_{\mathrm{i}} \mathrm{W}_{\mathrm{ij}} \mathrm{O}_{\mathrm{i}}+\mathrm{B}_{\mathrm{j}}\right)
$$

The transfer function $(\mathrm{F})$ can be sigmoidal or linear function. Generalized delta rule (GDR) is used for training of network with predefine threshold, learning rate, hidden node numbers, and maximum epoch

For RBF method, the mapping function is calculated according to following equation.

$$
\mathrm{Q}_{\mathrm{i}}=\Sigma_{i}^{\mathrm{m}} \mathrm{W}_{\mathrm{i}}^{*} \mathrm{~K}\left(\mathrm{i}_{\mathrm{u}} \ddot{\mathrm{S}}-\mathrm{S}_{\mathrm{i}} \mathrm{i} \ddot{\mathrm{u}}\right)
$$

Where $S$ is the tentative center and is one of input samples $S_{i}, i$ üS $-S_{i} i$ üis the Euclidean distance between $S$ and $S_{i} . K$ is the common radially symmetric kernel function, e.g. Gaussian function $\left(\mathrm{e}^{-(\mathrm{fmx}) 2}\right)$. Orthogonal Least Square (OLS) method is used for the learning of center positions with predefined spreading factor and error threshold.

Near infrared absorption spectra (reference to air) of 30 different concentrations are acquired from a spectrophotometer with the verification by a glucose analyzer (YSI-1500) before test. Two different normalization methods (1-exp(-OD) and Euclidean norm) are applied to the data set before the training or evaluating processes. The data set is then divided into odd and even groups according to its measured concentration value. We then subsequently used either one as calibration set and the other one as evaluation set. In cross validation, we used 29 spectra as the calibration set and the exclusive one for evaluation. The mean square error of evaluation set is calculated according to following equation:

$$
\text { MSE } \left.=£ U(\text { True Value }- \text { calculated Value })^{2} / n \quad i\right] 3 i^{\wedge}
$$

\section{RESULTS}

Table 1 lists the mean square error of RBF, BP and PLS methods. The simulation results show that with small number of samples, PLS can have better performance. However, this method becomes unreliable when the sample 
size growing too large. BP method can have comparable performance by using Euclidean Norm. BP can learn the maximum variations (principal components) embedded in samples. However, its performance degrades with the increasing number of samples. This can be caused by the limited storage capacity of fixed size network. RBF by its nature can dynamically increase the hidden nodes to accommodate the mapping function for desired accuracy. Thus, it is the only method, which achieves better performance with larger number of samples.

Table 1 Mean Square Error values of PLS, BP, and RBF

\begin{tabular}{|l|l|l|l|}
\hline & Odd/Even & Even/Odd & $\begin{array}{l}\text { Cross } \\
\text { validation }\end{array}$ \\
\hline \multicolumn{1}{|c|}{ RBF } & \multicolumn{3}{|c|}{} \\
\hline OD & 1048.2 & 795.4 & 711.9 \\
\hline 1 -exp(-OD) & 799.9 & 457.2 & 661.4 \\
\hline $\begin{array}{l}\text { Euclidean } \\
\text { Norm }\end{array}$ & 756.7 & 599.8 & 403.2 \\
\hline \multicolumn{1}{|c|}{ BP } & \multicolumn{3}{|c|}{} \\
\hline OD & 799.8 & 659.4 & 1262.9 \\
\hline 1 -exp(-OD) & 3284.4 & 32176 & 4358.9 \\
\hline $\begin{array}{l}\text { Euclidean } \\
\text { Norm }\end{array}$ & 595.8 & 461.4 & 704.4 \\
\hline \multicolumn{1}{|c|}{ PLS } & \multicolumn{3}{|l|}{} \\
\hline OD & 422.9 & 551.8 & 4916.7 \\
\hline 1 -exp(-OD) & 366.8 & 449.3 & 1789.8 \\
\hline $\begin{array}{l}\text { Euclidean } \\
\text { Norm }\end{array}$ & 529.5 & 649.6 & 4271.8 \\
\hline
\end{tabular}

\section{ACKNOWLEDGEMENT}

This work is supported by ITRI, R.O.C.

\section{REFERENCES}

[1] P. Bhandare, Y. Mendelson, R.A. Peura, G. Janatsch, J.D. Kruse-Jarres, R. Marbach, and H.M. Heise, "Multivariate determination of glucose in whole blood using partial least-squares and artificial neural networks based on mid-infrared spectroscopy," Appl. Spect. Vol. 47, pp.1214-1221, 1993.

[2] H.M. Heise, R. Marbach, T. Koschinsky, and F. A. Gries. "Noninvasive blood glucose sensors based on nearinfrared spectroscopy," Artif. Org. Vol.18, pp.439-447, 1994

[ 3 ] P.J. Gemperline, J.R. Long, and V.G. Gregoriou., "Nonlinear multivariate calibration using principal components regression and artificial neural networks," Anal. Chem. Vol.63, pp.2313-2323, 1991

[4] T.B., Blank and S.D. Brown, "Data processing using neural networks," Anal. Chim. Acta Vol. 277, pp.273-287, 1993

[5] C.-W.Lin, J.C. LaManna, and Y. Takefuji, "Quantitative Measurement of Two-Component pH- sensitive Colorimetric Spectra Using Multilayer Neural Networks," Biol. Cyber. Vol.67, pp.303-308, 1992

[6] T. Poggio and F. Girosi, "Networks for approximation and learning." Proc. of the IEEE, Vol.78, pp.1481-1497, 1990

[7] . J. Park, and I. W. Sandberg, "Universal approximation using radial-basis-function networks," Neural comput. Vol.3, pp.274-257,1991

[8] F. Girosi and T. Poggio, "Networks and the best approximation property". Biol. Cyber., Vol.63, pp. 169176,1990 